\title{
Aggregating Platelets Contract Isolated Canine Pulmonary Arteries by Releasing 5-Hydroxytryptamine
}

M. D. McGoon and P. M. Vanhoutte

Department of Physiology and Biophysics, and Division of

Cardiovascular Diseases, Mayo Clinic and Mayo Foundation, Rochester, Minnesota 55905 bstract. To examine the effect of platelets and 5-hydroxytryptamine on pulmonary arterial smooth muscle, rings of canine pulmonary arteries, with and without endothelium, were studied under isometric conditions in physiological salt solution. 5-Hydroxytryptamine, but not the thromboxane-like endoperoxide analogue U-46619, produced concentration-dependent contractions of the rings with a maximum averaging $93 \%$ of that obtained with $\mathrm{KCl}$. Autologous platelets in concentrations comparable to that in plasma caused contractions averaging $70 \%$ of the maximal responses to $\mathrm{KCl}$. Solution withdrawn from baths containing platelet-contracted rings, but not the supernatant from nonaggregated platelets, also caused contraction. The serotonergic antagonists cyproheptadine, ketanserin, and methysergide caused concentration-dependent inhibition and eventually abolition of contractions evoked by platelets and 5-hydroxytryptamine. Phentolamine and prazosin produced significantly less inhibition of the contractile response to platelets. Pretreatment of the platelets with indomethacin or meclofenamate reduced thromboxane release but had no effect on plateletinduced contractions. Removal of the endothelium did not affect contractile responses to platelets or 5-hydroxytryptamine. These experiments demonstrate that in the canine pulmonary artery: (a) 5-hydroxytryptamine is the predominant mediator of the contractile response triggered by platelet aggregation; and $(b)$ unlike in other blood vessels, the endothelium cannot curtail the contractile response to aggregating platelets.

A preliminary report of these findings was published in abstract form (1983. Fed. Proc. 42:593).

Received for publication 1 April 1983 and in revised form 9 August 1983.

J. Clin. Invest.

(c) The American Society for Clinical Investigation, Inc.

0021-9738/84/09/0828/06 $\$ 1.00$

Volume 74, September 1984, 828-833

\section{Introduction}

5-Hydroxytryptamine (serotonin) contracts canine and human isolated intrapulmonary vascular preparations $(1-4)$ and elevates pulmonary vascular resistance in intact organisms (5-10) or vascularly isolated perfused lungs (11-13). Microembolization or in situ aggregation of platelets produces an elevation of pulmonary vascular resistance, leading to pulmonary hypertension, which can be attributed in part to the release of vasoactive substances $(5,14-19)$. The present study was designed to answer the following questions: (a) Do aggregating platelets have a vasoconstrictive effect on isolated canine pulmonary arteries? (b) Does the release of 5-hydroxytryptamine contribute to platelet-evoked contractile responses? $(c)$ Do the vasoactive effects of 5-hydroxytryptamine or platelets depend upon the presence of the pulmonary vascular endothelium (20-22)?

\section{Methods}

The lower lobe of either lung was taken from adult mongrel dogs (15 to $30 \mathrm{~kg}$ ) after anesthesia with sodium pentobarbital $(30 \mathrm{mg} / \mathrm{kg}$ i.v.). After excision, the tissue was immersed in cold physiological salt solution of the following millimolar composition: $\mathrm{NaCl}, 118.3 ; \mathrm{KCl}$, 4.7; $\mathrm{CaCl}_{2}, 2.5 ; \mathrm{MgSO}_{4}, 1.2 ; \mathrm{KH}_{2} \mathrm{PO}_{4}, 1.2 ; \mathrm{NaHCO}_{3}, 25.0$; calcium disodium edetate, 0.026; glucose, 11.1. Segments of intrapulmonary artery $(\sim 4.0 \mathrm{~cm}$ long and 3.0 to $4.0 \mathrm{~mm}$ outside diameter) were dissected free, cleaned of adherent tissue, and cut into rings $(4.0 \mathrm{~mm}$ in length) without the touching of the luminal surface. In some preparations the endothelium was removed mechanically (20-22). Removal of endothelium was confirmed by the absence of the characteristic relaxation induced by acetylcholine of endothelium-intact rings contracted with norepinephrine (20-22).

The rings were mounted horizontally in organ baths filled with 25 $\mathrm{ml}$ of physiological salt solution $\left(37^{\circ} \mathrm{C}, \mathrm{pH} 7.4\right)$ aerated with $95: 5 \%$ $\mathrm{O}_{2} / \mathrm{CO}_{2}$. The preparations were connected to strain gauges for the recording of isometric force. The rings of pulmonary artery were stretched to the optimal point of their length-tension relationship by the use of $120 \mathrm{mM} \mathrm{KCl}$ (obtained by equimolar replacement of $\mathrm{NaCl}$ by $\mathrm{KCl}$ ). The rings were then allowed to equilibrate $30-60 \mathrm{~min}$. Responses of endothelium-denuded rings were compared with adjacent control rings only if the contractile response to $120 \mathrm{mM} \mathrm{KCl}$ of the endothelium-denuded ring was within $25 \%$ of that of the endotheliumintact ring.

Platelets. Autologous blood was collected in modified acid/citrate/ dextrose anticoagulant. Platelet-rich plasma was obtained by centrifugation $(180 \mathrm{~g}, 10 \mathrm{~min})$. A platelet pellet was obtained $(1600 \mathrm{~g}, 10 \mathrm{~min})$ 
and resuspended in calcium-free saline containing $0.4 \%$ citrate at $\mathrm{pH}$ 6.5 (23). Aliquots of this platelet suspension $(100 \mu \mathrm{l})$ were added to the organ chamber (volume reduced to $5 \mathrm{ml}$ for studies with platelets), resulting in a platelet concentration of $9.2 \pm 1.3 \times 10^{7} / \mathrm{ml}(n=16)$. Spontaneous platelet aggregation occurred after the addition of the suspensions to the physiological salt solution that contained the isolated rings. Aggregation was evidenced by a gradual clearing of the initially turbid solution, a visible platelet clumping, and scanning electron microscopy, which demonstrated platelet aggregates on the blood vessel (22). In some experiments, platelet suspensions were pretreated with indomethacin $\left(10^{-5} \mathrm{M}\right.$ for $\left.30 \mathrm{~min}\right)$ or meclofenamate $\left(10^{-5} \mathrm{M}\right.$ for 30 min); before addition to the organ chamber a platelet pellet was obtained again and resuspended, as described above.

Quantitation of thromboxane $B_{2}$. Samples $(n=5)$ of solution that contained aggregated platelets were withdrawn from the chamber after maximal tension of the ring had developed. After centrifugation $(3,000$ $g, 10 \mathrm{~min}$ ), the supernatant was frozen at $-20^{\circ} \mathrm{C}$ for up to $2 \mathrm{wk}$ until extraction. 2-ml aliquots of thawed supernatant were brought to $\mathrm{pH}$ 3.5 with $1 \mathrm{M}$ citric acid. Thromboxane $B_{2}$ was extracted twice with chloroform/methanol $(2: 1,6 \mathrm{ml})$. The organic solvents were evaporated to dryness in a $37^{\circ} \mathrm{C}$ water bath under a stream of nitrogen. $\left.100-\mu\right]$ aliquots of standards and diluted samples were assayed by displacement of $\left[{ }^{3} \mathrm{H}\right]$ thromboxane $B_{2}$ from thromboxane $B_{2}$-antiserum in a total incubation volume of $300 \mu \mathrm{l}$ at $4^{\circ} \mathrm{C}$. The reaction was stopped after 2 $h$ by the addition of a 1-ml solution of dextran-coated charcoal. After centrifugation $\left(3,000 \mathrm{~g}\right.$ at $4^{\circ} \mathrm{C}$ for $\left.5 \mathrm{~min}\right)$, the supernatant containing the antibody-bound $\left[{ }^{3} \mathrm{H}\right]$ thromboxane $B_{2}$ fraction was counted in a scintillation counter. The concentration of thromboxane $B_{2}$ was estimated by comparison with a standard curve. The lower limit of detection for this assay is $10 \mathrm{pg}$ of thromboxane $B_{2}(24)$.

Quantitation of amines. Supernatant was collected as described above and, after the addition of $4 \%$ cysteine $(100 \mu \mathrm{l} / \mathrm{ml})$ and $5 \%$ sodium metabisulfite $(10 \mu \mathrm{l} / \mathrm{ml})$, stored frozen at $-70^{\circ} \mathrm{C}$ for 2 wk. 5 Hydroxytryptamine, norepinephrine, and epinephrine concentrations were measured by high-pressure liquid chromatography with electrochemical detection (25). In brief, aliquots $(3 \mathrm{ml})$ of the supernatant were adjusted to $\mathrm{pH} 6.1$ and applied to columns $(2.3 \times 4 \mathrm{~mm})$ of the cation exchange resin Amberlite CG 50 (200-400 mesh). The amines were eluted and separation was by reversed-phase chromatography with a $\mu$ Bondpak $C_{18}$ column (Waters Associates, Milford, MA). The amines were quantitated by the injection of $25 \mu \mathrm{l}$ of their respective fractions onto the high-pressure liquid chromatography column and by the comparison of their peak height with those from a series of standard compounds. The limit of sensitivity (defined as signal-tonoise ratio > 5) was $12 \mathrm{pg}$ for each compound injected on the column. The column extraction procedure resulted in a fourfold concentration of 5-hydroxytryptamine and a threefold concentration of norepinephrine and epinephrine; thus, the limit of sensitivity was $125 \mathrm{pg}$ of 5hydroxytryptamine and $165 \mathrm{pg}$ of norepinephrine or epinephrine per ml of supernatant.

Drugs. The following drugs were used: cyproheptadine $\mathrm{HCl}, 5-$ hydroxytryptamine creatinine sulfate, indomethacin, acetylcholine chloride, L-arterenol bitartrate, and norepinephrine (Sigma Chemical Company, St. Louis, MO); rabbit antithromboxane $\mathbf{B}_{2}$ (Seragen, Inc., Boston, MA); absolute methanol and analytic-grade chloroform (J. T. Baker Chemical Co., Phillipsburg, NJ); endoperoxide analogue U46619, 15 S-hydroxy-11 $\alpha, 9 \alpha$-(epoxymethano)prosta-5Z 13E-dienoic acid (Upjohn Co., Kalamazoo, MI); ketanserin tartrate (Janssen Pharmaceutica, Beerse, Belgium); sodium meclofenamate (Parke, Davis and Co., Detroit, MI); methysergide maleate (Sandoz Pharmaceutical
Div., Hanover, NJ); sodium pentobarbital (Fort Dodge Laboratories, Fort Dodge, IA); phentolamine mesylate (Ciba-Geigy Corp., Pharmaceuticals Div., Summit, NJ); prazosin $\mathrm{HCl}$ (Pfizer Chemicals Div., Pfizer, Inc., NY); scintillation fluid (Safety Solve; Research Products International Corp., Mount Prospect, $\mathrm{IL}$ ); and $\left[{ }^{3} \mathrm{H}\right]$ thromboxane $\mathrm{B}_{2}$, $163 \mathrm{Ci} / \mathrm{mM}$ (New England Nuclear, Boston, MA). Solutions of all drugs used in organ chamber experiments were prepared daily, kept on ice, and added to the organ chamber in $100-\mu$ l aliquots. Concentrations of drugs are expressed as final molar bath concentrations (M). Stock solution of indomethacin was prepared with equimolar amounts of $\mathrm{Na}_{2} \mathrm{CO}_{3}$ immediately before use. Prazosin was dissolved in $100 \mu \mathrm{l}$ of dimethylsulfoxide before it was added to $10 \mathrm{ml}$ of distilled water to make the stock solution. Methysergide was dissolved in $100 \mu l$ of ethanol before the addition of $10 \mathrm{ml}$ of distilled water. Solvent controls were performed in parallel when dimethylsulfoxide or ethanol was used. All other drugs were dissolved in distilled water.

Calculations and statistical analysis. In each experimental group, the number of rings equals the number of animals studied. To correct for changes in sensitivity with time, control preparations obtained from the same vessels as those exposed to an experimental agent were studied simultaneously. Data are expressed as means \pm SEM. In certain experiments, cumulative concentration-response curves to agonists were obtained by the cumulative increasing of bath concentrations approximately threefold (26). To assess the potency of antagonists, concentration-response curves to 5-hydroxytryptamine or norepinephrine were obtained in the absence and in the presence of increasing concentrations of the antagonists. In some experiments, the inhibitory potency of antagonists was evaluated by their ability to relax vessels previously contracted by 5-hydroxytryptamine or platelets. The concentration of drug was increased only after the previous concentration had produced a stable response.

Statistical analysis was performed by the $t$ test for paired or unpaired observations (two tailed), or by analysis of variance. Paired observations were considered to be those involving the same vessel ring before and after intervention. $P<0.05$ was considered significant.

\section{Results}

Platelets. The addition of platelets to the organ chamber caused contractions of all pulmonary arteries from 16 dogs. The contractions $(70 \pm 18 \%$ of the increase in tension obtained with $120 \mathrm{mM} \mathrm{KCl}$ ) were sustained for as long as $30 \mathrm{~min}$ and were reversible upon washing out the platelets (Fig. $1 A$ ). The supernatant of platelet suspensions (obtained by centrifugation at $1,600 \mathrm{~g}$ for $10 \mathrm{~min}$ ) had no effect (Fig. $1 \mathrm{~A}$ ). When added to organ chambers that contained quiescent preparations, the supernatant $(1,600 \mathrm{~g}$ for $10 \mathrm{~min})$ of physiological salt solution withdrawn from organ chambers containing rings contracted by platelets evoked contractions comparable to those produced by the aggregating platelets $(n=4$; Fig. $1 B$ ). The concentration of 5-hydroxytryptamine in the supernatant was $5.3 \pm 1.0$ $\times 10^{-7} \mathrm{M}(n=6)$, and the norepinephrine concentration was $8.7 \pm 5.4 \times 10^{-9} \mathrm{M}(n=5)$. Epinephrine was not detected in four of five samples $\left(2.9 \times 10^{-9} \mathrm{M}\right.$ in one supernatant). The pretreatment of platelet suspensions with indomethacin (1 $\times 10^{-5} \mathrm{M}$ for $\left.30 \mathrm{~min} ; n=6\right)$ or meclofenamate $\left(1 \times 10^{-5} \mathrm{M}\right.$ for $30 \mathrm{~min} ; n=5$ ) did not affect contractile responses to platelets added to the organ chamber (Fig. $1 C$ ). The concen- 


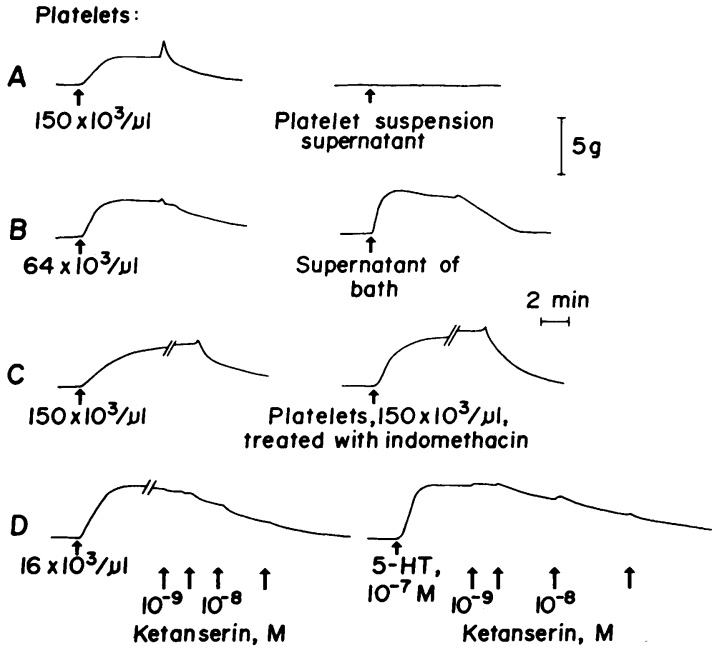

Figure 1. Recordings of isometric tension in pulmonary arterial rings from four dogs. $(A)$ Exposure to platelets is followed by prominent, sustained contractions which reversed upon washing; supernatant from suspensions of nonaggregated platelets produced no response. $(B)$ Supernatant of bath solution containing a ring contracted by platelets elicits a comparable response when re-added to the organ chamber. $(C)$ Pretreatment of platelets with indomethacin does not alter the contractile response to equal concentrations of platelets. $(D)$ Contractions of comparable magnitude produced by platelets or 5 hydroxytryptamine were reversed similarly by ketanserin. Horizontal bar depicts time; vertical bar represents tension in grams. All responses were compared with those of simultaneous time-controls (not shown).

tration of thromboxane $B_{2}$ (the stable hydrolysis product of thromboxane $A_{2}$ ) in the bath was significantly decreased by pretreatment with meclofenamate (from $6.5 \pm 2.5$ to $2.2 \pm 1.9$ $\mathrm{ng} / \mathrm{ml})$
Cyproheptadine, ketanserin, and methysergide caused concentration-dependent inhibition of the platelet-evoked contractions (Fig. $2 \mathrm{~A}$ ). Phentolamine and prazosin caused concentration-dependent reductions of the response to aggregating platelets; at $10^{-7} \mathrm{M}$, the inhibitory effect of either alpha-adrenergic antagonist was significantly less than that of any serotonergic inhibitor (Fig. $2 A$ ).

5-Hydroxytryptamine. 5-Hydroxytryptamine caused concentration-dependent $\left(10^{-8}\right.$ to $\left.3 \times 10^{-6} \mathrm{M}\right)$ contraction of pulmonary arterial rings $(n=12)$. The maximal contraction averaged $93 \pm 20 \%$ of that produced by $20 \mathrm{mM} \mathrm{KCl}$. Ketanserin $\left(10^{-9}\right.$ to $\left.10^{-7} \mathrm{M}\right)$, but not phentolamine $\left(10^{-9}\right.$ to $\left.10^{-6} \mathrm{M}\right)$, caused a parallel shift to the right of the concentration-response curve to 5-hydroxytryptamine (Fig. 3). In rings contracted with a concentration of 5-hydroxytryptamine that caused comparable increases in tension as aggregating platelets, the addition of cumulative concentrations of ketanserin produced similar relaxations of each ring (Figs. $1 D$ and $2 B$ ).

Norepinephrine. Norepinephrine caused concentration-dependent contraction of pulmonary artery rings. The maximal contraction averaged $127 \pm 20 \%$ of that caused by $120 \mathrm{mM}$ $\mathrm{KCl}$. Phentolamine caused a parallel shift to the right of the concentration-response curve to norepinephrine (Fig. 3). Ketanserin $\left(10^{-9}\right.$ to $\left.10^{-6} \mathrm{M}\right)$ also produced a parallel shift to the right of the concentration-response curve to norepinephrine (Fig. 3); the shift was smaller and occurred at higher concentrations of ketanserin than did those required to affect the response to 5-hydroxytryptamine.

$U-46619$. The endoperoxide analogue U-46619 $\left(1.0 \times 10^{-6}\right.$ M) did not cause significant increases in tension $(n=5)$, whereas the platelet evoked contractions in these vessels averaged $2.1 \pm 0.3 \mathrm{~g}$.

Removal of endothelium. The removal of the endothelium from pulmonary arterial $(n=6)$ rings did not significantly affect the magnitude of the responses of pulmonary artery
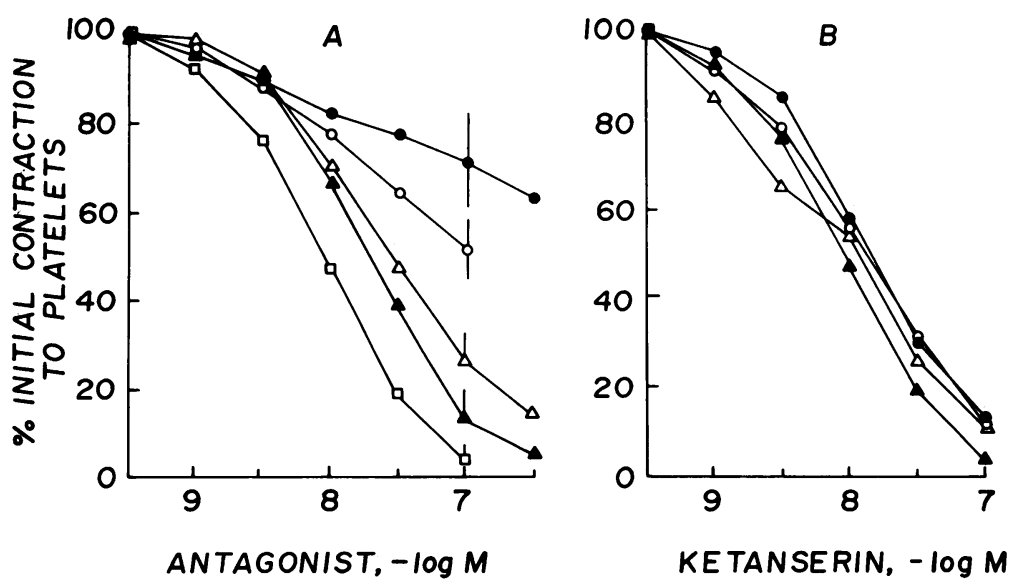

Figure 2. (A) Concentration-response curves of various serotonergic- or alpha-adrenoceptor antagonists on pulmonary arterial rings constricted by addition of platelets; the difference in relaxation produced by $10^{-7} \mathrm{M}$ of either alpha-antagonist is significantly less than that obtained at equal concentration of any serotonergic-antagonist $(P$ $<0.05$ ). The antagonists used were phentolamine (O), prazosin $(\bullet)$, methysergide $(\Delta)$, cyproheptidine $(\Delta)$, and ketanserin ( $\square$ ). (B) Concentration-response curves of ketanserin on endothelium-intact and endothelium-denuded rings contracted by platelets or 5-hydroxytryptamine. Vessels (with [•] or without [o] endothelium) were contracted by 5 -hydroxytryptamine. Vessels (with [ [ ] or without $[\Delta]$ endothelium) were contracted by platelets. Tension at $100 \%$ of platelet-evoked contraction was $2.8 \pm 0.5 \mathrm{~g}$, and for 5-hydroxytryptamine-induced contractions it was $3.6 \pm 0.6 \mathrm{~g}$. Percentage of initial contraction at each concentration of antagonist is shown as the mean for all vessels tested. For the sake of clarity, SEMs are omitted. 


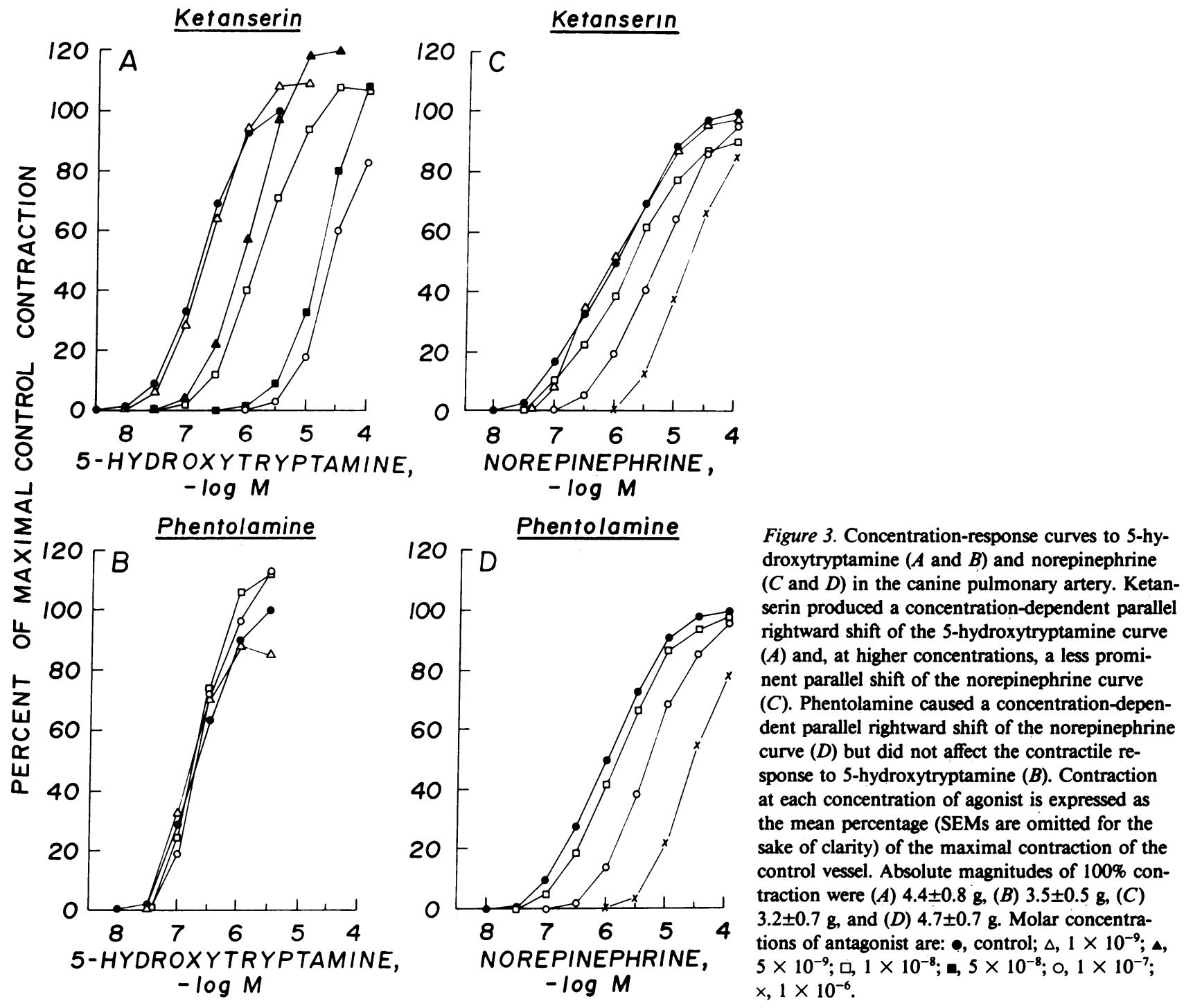

rings to either platelets, 5-hydroxytryptamine, or norepinephrine. These averaged, respectively, $103 \pm 21,130 \pm 33$, and $107 \pm 14 \%$ of the contractions obtained in endothelium-intact rings.

\section{Discussion}

The present study confirms previous reports (1-3) that 5hydroxytryptamine is a potent constrictor of the canine pulmonary artery and produces maximal contractile responses comparable to those obtained with $\mathrm{KCl}$ solution or norepinephrine. It demonstrates that the exposure of platelets to isolated pulmonary artery rings contained in physiological saline causes aggregation of the platelets, release of platelet products, and contraction of the vascular smooth muscle. The response produced by platelets is mediated primarily by the release of 5-hydroxytryptamine since serotonergic antagonists reverse the platelet-evoked contractile response. The conclusions of this study agree with previous investigations of plateletinduced contraction of rat caudal artery preparations (27) in which ketanserin, but not cyclooxygenase blockade, markedly inhibited contractions caused by platelets. In the rat caudal artery, however, relaxation by ketanserin of platelet-contracted vessels was not complete and, unlike that observed in the present study, did not parallel precisely the relaxation of vessels contracted with 5-hydroxytryptamine.

Other products of platelet aggregation, including catecholamines or thromboxane $A_{2}$, may produce pulmonary vascular contraction (28-31). The failure of cyclooxygenase inhibitors to alter the contractile response to platelets indicates, however, that thromboxane $A_{2}$ is not an essential mediator of plateletevoked contraction of the canine pulmonary artery. This conclusion is supported by absence of a contractile response 
to the thromboxane-like (32) endoperoxide analogue U-46619 in concentrations 100 -fold greater than the thromboxane $B_{2}$ level observed after platelet aggregation.

That alpha-adrenergic antagonists partially reversed the contraction induced by platelets cannot be attributed easily to serotonergic blockade, since phentolamine in the concentrations used did not produce a rightward shift of the concentrationresponse curve to 5-hydroxytryptamine. Since the relaxation produced by alpha-adrenergic blockade was greater than the residual platelet-evoked contraction after serotonergic blockade, part of the inhibition caused by serotonergic antagonists could be the consequence of an interaction with alpha-adrenoceptors. In the case of ketanserin, the nearly complete relaxation it caused may be due to superimposed alpha-adrenergic blocking properties of the drug (33), but this explanation is unlikely in view of the high concentrations required to affect the contractile response to norepinephrine. Furthermore, this interpretation does not apply to cyproheptadine and methysergide. The present experiments demonstrate that the aggregating platelets release small amounts of catecholamines, in particular norepinephrine. A possible explanation for the observed effects of alpha-adrenergic and serotonergic inhibitors is that the released 5-hydroxytryptamine not only causes direct activation of the vascular smooth muscle but also amplifies its response to the catecholamines released from the platelets $(33,34)$. The amplifying effect of 5-hydroxytryptamine is antagonized by serotonergic blockers $(33,34)$.

The endothelial cells can mediate a variety of inhibitory responses of the vascular smooth muscle of the media $(20,21$, $22,35)$. Thus, in the canine coronary artery, the presence of endothelial cells effectively reduces contractions evoked by aggregating platelets (22). In the present study, the preparations with intact endothelium reacted as expected to acetylcholine (21), whereas those denuded of endothelial cells did not. However, the contractile responses of the pulmonary vascular smooth muscle to either platelets or 5-hydroxytryptamine are not influenced by the integrity of the endothelium, providing a new, striking example of heterogeneity of endothelial responsiveness (22).

Insofar as data obtained on isolated pulmonary arteries can be extrapolated to the intact organism, the present observations suggest that 5-hydroxytryptamine is indeed the predominant mediator of the pulmonary vasoconstriction caused by aggregating platelets $(5,14-17,19)$. This effect of 5hydroxytryptamine might play a role in pulmonary hypertension and is likely to contribute to the vasoconstrictions during pulmonary embolism (36). The fact that, unlike in coronary vessels, the pulmonary endothelial cells cannot curtail the contractile response to aggregating platelets may be particularly important in maintaining spastic constrictions of large pulmonary arteries upon embolization.

\section{Acknowledgments}

The authors are grateful to Dr. Silvia Romero, Department of Physiology, for determination of thromboxane $B_{2}$ levels and to Dr. Gertrude
Tyce, Department of Physiology, for measurement of 5-hydroxytryptamine, norepinephrine, and epinephrine concentrations. The authors also thank Mr. Robert Lorenz for preparing the illustrations and Mrs. Janet Beckman for excellent secretarial assistance.

Dr. McGoon was supported in part by the American College of Cardiology/Merck Training Award for 1982-1983. This study was supported in part by grant HL 21584 of the National Institutes of Health and by the Mayo Foundation.

\section{References}

1. Joiner, P. D., P. J. Kadowitz, L. B. Davis, and A. L. Hyman. 1975. Contractile responses of canine isolated pulmonary lobar arteries and veins to norepinephrine, serotonin and tyramine. Can. J. Physiol. Pharmacol. 53:830-838.

2. Chand, N., and B. M. Altura. 1980. Serotonin receptors subserve only contraction in canine and rat pulmonary arteries and veins. Artery. 7:232-245.

3. Gruetter, C. A., L. J. Ignarro, A. L. Hyman, and P. J. Kadowitz. 1981. Contractile effects of 5-hydroxytryptamine in isolated intrapulmonary arteries and veins. Can. J. Physiol. Pharmacol. 59:157-162.

4. Freeman, W. K., D. K. Rorie, and G. M. Tyce. 1981. Effects of 5-hydroxytryptamine on neuroeffector junction in human pulmonary artery. J. Appl. Physiol. 51:693-698.

5. Comroe, J. H., B. Van Lingen, R. C. Stroud, and A. Roncoroni. 1953. Reflex and direct cardiopulmonary effects of 5-OH-tryptamine (serotonin). Am. J. Physiol. 173:379-386.

6. Borst, H. G., E. Berglund, and M. McGregor. 1957. The effects of pharmacologic agents on the pulmonary circulation of the dog. Studies on epinephrine, norepinephrine, 5-hydroxytryptamine, acetylcholine, histamine, and aminophylline. J. Clin. Invest. 36:669-675.

7. Shepherd, J. T., D. E. Donald, E. Linder, and H. J. C. Swan. 1959. Effect of small doses of 5-hydroxytryptamine (serotonin) on pulmonary circulation in the closed-chest dog. Am. J. Physiol. 197:963967.

8. McGaff, C. J., and W. R. Milnor. 1962. Effects of serotonin on pulmonary blood volume in the dog. Am. J. Physiol. 202:957-960.

9. Sackner, M. A., D. H. Will, and A. B. Dubois. 1966. The site of pulmonary vasomotor activity during hypoxia or serotonin administration. J. Clin. Invest. 45:112-121.

10. Levy, S. E., and D. H. Simmons. 1971. Serotonin, pulmonary hypertension, and airway constriction in the anesthetized dog. Proc. Soc. Exp. Biol. Med. 138:365-368.

11. Gilbert, R. P., L. B. Hinshaw, H. Kuida, and M. B. Visscher. 1958. Effects of histamine, 5-hydroxytryptamine and epinephrine on pulmonary hemodynamics with particular reference to arterial and venous segment resistances. Am. J. Physiol. 194:165-170.

12. Rickaby, D. A., C. A. Dawson, and M. B. Maron. 1980. Pulmonary inactivation of serotonin and site of serotonin pulmonary vasoconstriction. J. Appl. Physiol. 48:606-612.

13. Bhattacharya, J., S. Nanjo, and N. C. Staub. 1982. Micropuncture measurement of lung microvascular pressure during 5-HT infusion. $J$. Appl. Physiol. 52:634-637.

14. Hyland, J. W., T. E. Piemme, S. Alexander, F. W. Haynes, G. T. Smith, and L. Dexter. 1963. Behavior of pulmonary hypertension produced by serotonin and emboli. Am. J. Physiol. 205:591-597.

15. Rosoff, C. B., E. W. Salzman, V. Gurewich, and H. K Schroeder. 1971. Reduction of platelet serotonin and the response to pulmonary emboli. Surgery (St. Louis). 70:12-19. 
16. Woolverton, W. C., and A. L. Hyman. 1973. The pulmonary hemodynamic effects of lung thromboemboli in dogs. Surgery (St. Louis). 73:572-578.

17. Ozdemir, I. A., W. R. Webb, and S. D. Wax. 1974. Effect of neural and humoral factors on pulmonary hemodynamics and microcirculation in pulmonary embolism. J. Thorac. Cardiovasc. Surg. 68:896-904.

18. Melczoch, J., A. Tucker, E. K. Weir, J. T. Reeves, and R. F. Grover. 1978. Platelet-mediated pulmonary hypertension and hypoxia during pulmonary microembolism. Reduction by platelet inhibition. Chest. 74:648-653.

19. Utsunomiya, T., M. M. Krausz, D. Shepro, and H. B. Hechtman. 1981. Prostaglandin control of plasma and platelet 5-hydroxytryptamine in normal and embolized animals. Am. J. Physiol. 241:H766-H771.

20. Furchgott, R. F., and J. V. Zawadzki. 1980. The obligatory role of endothelial cells in the relaxation of arterial smooth muscle by acetylcholine. Nature (Lond.). 288:373-376.

21. De Mey, J. G., and P. M. Vanhoutte. 1982. Heterogeneous behavior of the canine arterial and venous wall: importance of the endothelium. Circ. Res. 51:439-447.

22. Cohen, R. A., J. T. Shepherd, and P. M. Vanhoutte. 1983. Inhibitory role of the endothelium in the response of isolated coronary arteries to platelets. Science (Wash. DC). 221:273-274.

23. Dewanjee, M. K., S. A. Rao, and P. Didisheim. 1981. Indium111 tropolone, a new high-affinity platelet label: preparation and evaluation of labeling parameters. J. Nucl. Med. 22:981-987.

24. Strand, J. C., B. S. Edwards, M. E. Anderson, J. C. Romero, and F. G. Knox. 1981. Effect of imidazole on renal function in unilateral ureteral-obstructed rat kidneys. Am. J. Physiol. 240:F508F514.

25. Tyce, G. M., and T. L. Yaksh. 1981. Monoamine release from cat spinal cord by somatic stimuli: an intrinsic modulatory system. $J$. Physiol. 314:513-529.

26. Van Rossum, J. M. 1963. Cumulative dose-response curves. II. Technique for the making of dose-response curves in isolated organs and the evaluation of drug parameters. Arch. Int. Pharmacodyn. Ther. 143:299-330.
27. De Clerck, F., and J. M. Van Nueten. 1982. Platelet-mediated vascular contractions: inhibition of the serotonergic component by ketanserin. Thromb. Res. 27:713-727.

28. Altura, B. M., and N. Chand. 1981. Differential effects of prostaglandins on canine intrapulmonary arteries and veins. $B r$. $J$. Pharmacol. 73:819-827.

29. Kadowitz, P. J., C. A. Gruetter, E. W. Spannhake, and A. L. Hyman. 1981. Pulmonary vascular responses to prostaglandins. Fed. Proc. 40:1991-1996.

30. Boyd, J. A., and T. E. Eling. 1980. Prostaglandin release and the interaction of platelets with the pulmonary vasculature of rat and guinea pig. Thromb. Res. 19:239-248.

31. Vaage, J. 1982. Intravascular platelet aggregation and pulmonary injury. Ann. NY Acad. Sci. 384:301-318.

32. Coleman, R. A., P. P. A. Humphrey, I. Kennedy, G. P. Levy, and P. Lumley. 1981. Comparison of the actions of U-46619, a prostaglandin $\mathrm{H}_{2}$-analogue, with those of prostaglandin $\mathrm{H}_{2}$ and thromboxane $A_{2}$ on some isolated smooth muscle preparations. $B r . J$ Pharmacol. 73:773-778.

33. Van Nueten, J. M., P. A. J. Janssen, J. Van Beek, R. Xhonneux, T. J. Verbeuren, and P. M. Vanhoutte. 1981. Vascular effects of ketanserin ( $R$ 41 468), a novel antagonist of 5-HT 2 serotonergic receptors. J. Pharmacol. Exp. Ther. 218:217-230.

34. Van Nueten, J. M., P. A. J. Janssen, W. De Ridder, and P. M. Vanhoutte. 1982. Interaction between 5-hydroxytryptamine and other vasoconstrictor substances in the isolated femoral artery of the rabbit: effect of ketanserin (R 41 468). Eur. J. Pharmacol. 77:281-287.

35. De Mey, J. G., M. Claeys, and P. M. Vanhoutte. 1982 Endothelium-dependent inhibitory effects of acetylcholine, adenosine triphosphate, thrombin and arachidonic acid in the canine femoral artery. J. Pharmacol. Exp. Ther. 222:166-173.

36. Huval, W. V., M. A. Mathieson, I. I. Stemp, B. M. Dunham, A. G. Jones, D. Shepro, and H. B. Hechtman. 1983. Therapeutic benefits of 5-hydroxytryptamine inhibition following pulmonary embolism. Ann. Surg. 197:220-225. 